\title{
Research Paper \\ Effect of Aerobic Exercise With Blood Flow Restriction on Mitochondrial Dynamics Proteins of Human Skeletal Muscles
}

\author{
Ali Aryashakib ${ }^{1}$ (D), *Bahman Mirzaei ${ }^{1}$ (1), Payam Saidie ${ }^{1}$ (1)
}

1. Department of Exercise Physiology, Faculty of Physical Education and Sport Sciences, University of Guilan, Rasht, Iran.

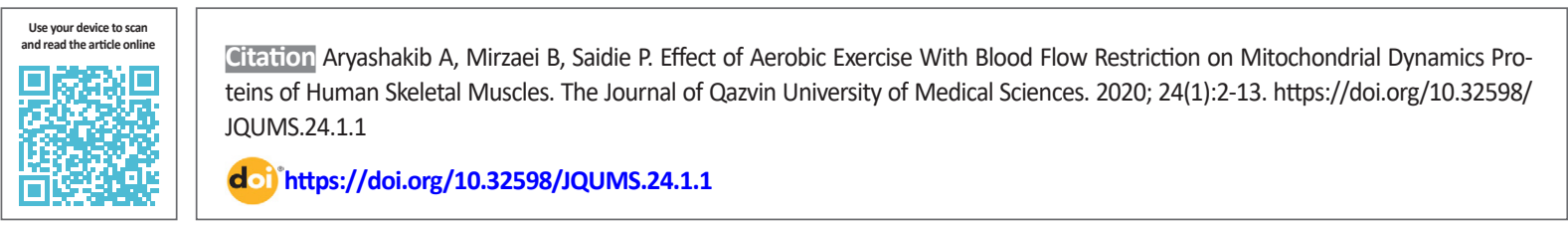

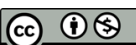

Received: 20 Dec 2018

Accepted: 23 Apr 2019

Available Online: 01 Apr 2020

Keywords:

Aerobic exercise, Blood flow restriction, Mitochondrial dynamics, Dynaminrelated protein 1 Mitofusin 2

\section{ABSTRACT}

Background Aerobic exercise with Blood Flow Restriction (BFR) plays an important role in skeletal muscle adaptation; however, the effects of this type of exercise on mitochondrial dynamics proteins are unclear. Objective The purpose of this study was to investigate the effect of aerobic exercise with and without BFR on mitochondrial dynamics proteins of human skeletal muscles.

Methods Participants were 5 young men (mean age, 33.4 \pm 2.30 years; mean weight, $79.64 \pm 10.49 \mathrm{~kg}$; BMI, $26.24 \pm 2.27 \mathrm{~kg} / \mathrm{m}^{2}$ ). They performed aerobic exercise with BFR (AE+BFR) and without BFR (AE) in two separate days at five 2-min sessions and 1 min rest between the sessions. Western Blot method was used to measure the protein levels of Mitofusin 2 (MFN2) and Dynamin-Related Protein 1 (DRP1) in skeletal muscles.

Findings $A E+B F R(1.02 \pm 0.05$ vs. $0.77 \pm 0.03)$ and $A E(0.65 \pm 0.08$ vs $0.57 \pm 0.03)$ significantly increased the mean MFN2 protein level compared to the pre-test mean values $(P<0.05)$. $A E+B F R(3.54 \pm 0.46$ and $5.01 \pm 0.66)$ and $A E(3.38 \pm 0.38$ vs. $2.82 \pm 0.59)$ also significantly reduced the mean $D R P 1$ level $(P<0.05)$. Moreover, $A E+B F R$ had greater significant effect on the mean levels of MFN2 $(0.24 \pm 0.01$ vs. $0.08 \pm 0.04)$ and DRP1 ( $-1.46 \pm 0.22$ vs. $-0.33 \pm 0.12)$ compared to $A E(P<0.05)$.

Conclusion It seems that aerobic exercise with BFR is a strong stimulant for the improvement of skeletal muscle mitochondrial dynamics.

\section{Extended Abstract}

\section{Introduction}

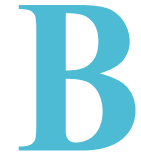

lood Flow Restriction (BFR) as a new training method has been increasingly used to apply more physiological stress with lowintensity exercise. Exercise with BFR reduces oxygen delivery to skeletal muscle as well as the clearance of produced metabolites. It creates a stressful muscular environment that may be a strong stimulus for physiological adaptations [1]. However, the effect of BFR training with aerobic exercise on aerobic performance-related parameters has been received less attention. Changes and adaptations caused by exercise on muscle mitochondria can occur as a result of mitochondrial biogenesis, mitochondrial dynamics (including fusion and fission), and the process of mitophagy which can be an important mechanism for improving muscle oxygen consumption and, consequently, athletic performance [8].

Mitochondrial fusion plays an important role in maintaining mitochondrial integrity and is dependent on mitofusin (MFN) 1 and 2 [9], while during mitochondrial fission,

\section{* Corresponding Author:}

Bahman Mirzaei

Address: Department of Exercise Physiology, Faculty of Physical Education and Sport Sciences, University of Guilan, Rasht, Iran.

Tel: +98 (912) 32157778

E-Mail: mirzaei@fila-wrestling.com 
mitochondrial fragmentation expands and is dependent on Dynamin-Related Protein 1 (DRP1) [11]. Despite the effective role of exercise with BFR, there is no study of the potential role of this type of activity on mitochondrial fusion and fission proteins. Therefore, due to this limitation, the aim of this study was to investigate the effect of aerobic exercise with and without BFR on mitochondrial dynamic proteins (MFN2 and DRP1) of human skeletal muscle.

\section{Materials and Methods}

This is a quasi-experimental cross-sectional study with pre-test/post-test design. Participants were 5 male students at University of Guilan (Mean \pm SD age, $33.40 \pm 2.30$ years; Mean \pm SD weight, $79.64 \pm 10.49 \mathrm{~kg}$; BMI, $26.24 \pm 2.27 \mathrm{~kg} / \mathrm{m}^{2}$ ) who were selected using a convenience sampling method. They performed aerobic exercise with BFR (AE+BFR) and aerobic exercise (AE) without BFR in 2 days. In $A E+B F R$ intervention, BFR was applied by the pressure cuff on the proximal thigh area, and then, after warming up (including walking, running, and stretching), the subjects began to walk (with a speed of 51 meters per minute) on the treadmill.

The walking program included five 2-minute sessions and 1 minute rest between each session, according to Takashi et al. [20]. BFR in the leg muscles was maintained throughout the training session and 1-min rest interval (for 14 minutes which reached 17 minutes with 3-min warming up). The BFR was lifted immediately after the fifth session of walking, and then a period of return to initial state, including
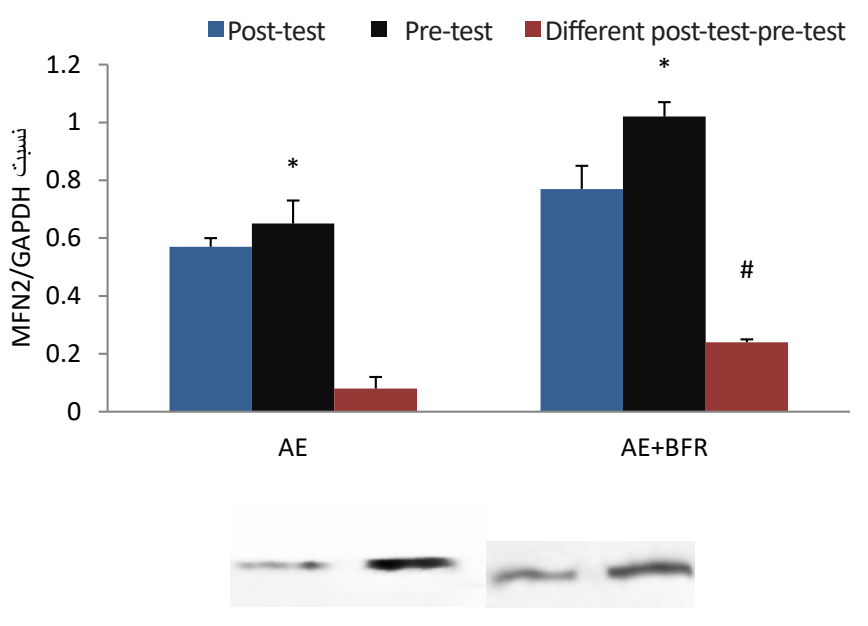

Qazvin University of Medical Sciences (JOUMS)

Figure 1. Effect of AE+BFR and AE on the MFN2 protein content (MFN2/GAPDH ratio), and western blot analysis of the expressions of MFN2 and GAPDH.* Significant compared to the pre-test phase $(\mathrm{P}<0.05)$, \# significant compared to $\mathrm{AE}(\mathrm{P}<0.05)$
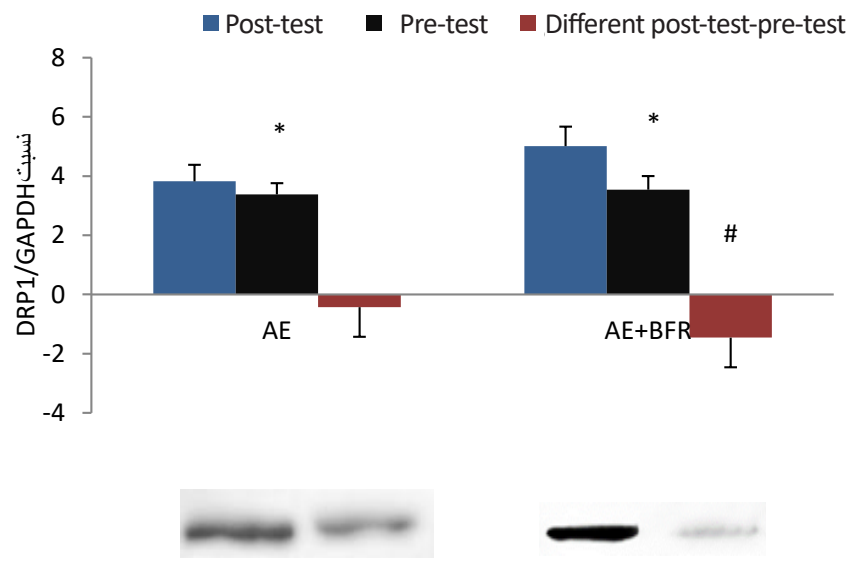

Figure 2. Effect of AE $+B F R$ and AE on the DRP1 protein content (DRP1/GAPDH ratio), and western blot analysis of the expressions of MFN2 and GAPDH.* Significant compared to the pre-test phase $(\mathrm{P}<0.05)$; \# significant compared to $\mathrm{AE}(\mathrm{P}<0.05)$ 
walking, was performed by subjects for 5-11 minutes. $\mathrm{AE}$ intervention was similar to BFR+BFR, but was performed without applying BFR. In order to evaluate the changes in MFN2 and DRP1, a biopsy of the lateral extensor muscle was performed in two stages: 5 minutes before the start of both exercise interventions and 3 hours after their completion. Western blotting was used to measure the protein values of MFN2 and DRP1. In order to determine the difference between pre-test and post-test scores and also the difference between scores of two interventions, the paired t-test was used at the significance level of 0.05 .

\section{Results}

The results showed that both aerobic exercise with BFR ( $\mathrm{P}=0.001)$ and without BFR ( $\mathrm{P}=0.04)$ led to a significant increase in MFN2 protein value compared to the pre-test values. A comparison between the two interventions showed that $\mathrm{AE}+\mathrm{BFR}$ led to a significant increase in MFN2 protein value compared to $\mathrm{AE}$ $(\mathrm{P}=0.01)$ (Figure 1). The results also showed that both $\mathrm{AE}+\mathrm{BFR}(\mathrm{P}=0.001)$ and $\mathrm{AE}(\mathrm{P}=0.02)$ led to a significant increase in DRP1 protein value compared to the pre-test values. A comparison between the two interventions showed that $\mathrm{AE}+\mathrm{BFR}$ also could significantly increase the DRP1 protein value compared to AE $(\mathrm{P}=0.003)$ (Figure 2).

\section{Discussion}

Although there is very limited information on the acute effects of aerobic exercise on mitochondrial dynamics proteins, the findings of the present study showed that aerobic exercise can increase mitochondrial dynamics by reducing fission and increasing fusion, regardless of the role of BFR. Due to the role of PGC- $1 \alpha$ in the regulation of proteins involved in mitochondrial fusion and fission, and the upregulation of PGC-1 $\alpha$ activity, both aerobic exercise with and without BFR could increase MFN2 level and decrease DRP1 level by up-regulation of PGC-1 $\alpha$ activity [25].

Although there is no direct evidence to compare the effect of exercise with and without BFR on PGC- $1 \alpha$ signaling and its regulators in the present study, higher induction and stimulation of PGC- $1 \alpha$ following exercise with BFR seems to have resulted in further stimulation of mitochondrial dynamics proteins. Moreover, it is possible that aerobic exercise with BFR leads to an optimal increase in Reactive Oxygen Species (ROS) and PGC-1 $\alpha$ stimulation, followed by improved mitochondrial dynamics three hours before exercise [30, 31]. Overall, it was concluded that aerobic exercise with BFR can facilitate mitochondrial dynamics by increasing both mitochondrial fusion and fission processes.

\section{Ethical Considerations}

\section{Compliance with ethical guidelines}

This study obtained its ethical approval from the Research Ethics Committee of Guilan University of Medical Sciences (Code: IR.GUMS.REC.1397.061).

\section{Funding}

This study was extracted from the $\mathrm{PhD}$. thesis of the first author in Department of Exercise Physiology, Faculty of Physical Education and Sport Sciences, University of Guilan, Rasht.

\section{Authors' contributions}

Conceptualization and validation: Ali Aryashakib and Bahman Mirzaei; Methodology, draft preparation, data analysis: All authors; Resources and funding acquisition: Ali Aryashakib; Editing and review: Bahman Mirzaei and Payam Saidie; Supervision and project administration: Bahman Mirzaei.

\section{Conflicts of interest}

The authors declared no conflict of interest

\section{Acknowledgements}

The authors would like to thank participants and the Faculty of Physical Education and Sports Sciences at University of Guilan for their cooperation. 


\title{
تأثير فعاليت ورزشى هوازى با محدوديت جريان خون بر بروتئينهاى ديناميك ميتوكندرى عضله اسكلتى انسان
}

\author{
على آرياشكيب' هـ، "بهمن ميرزايى' هـ ييام سعيدى' \\ اكروه فيزيولوزي ورزشى مانشكده تربيتبدنى و علوم ورزشى، دانشعاه كيلان، ايران.
}

\begin{abstract}
(1)

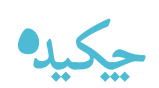

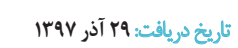

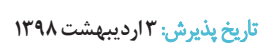

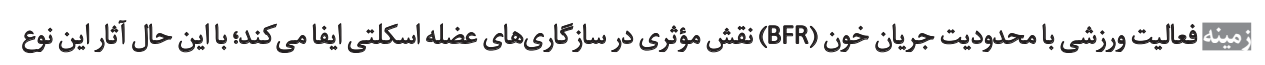

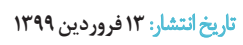

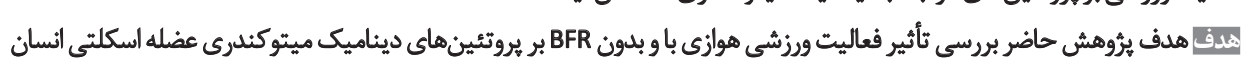

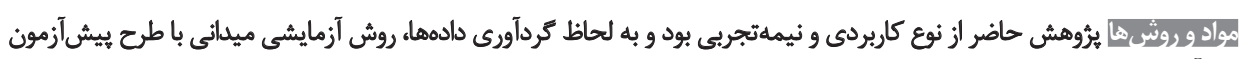

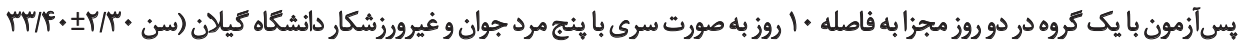

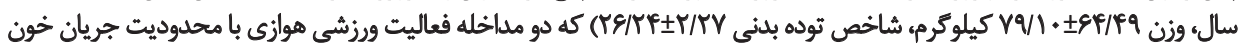

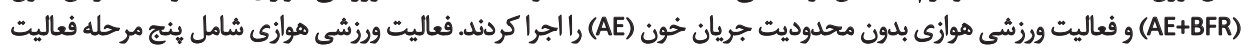

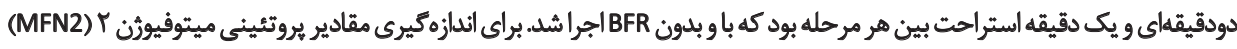

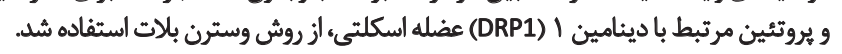

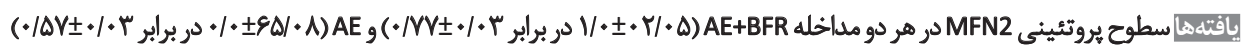

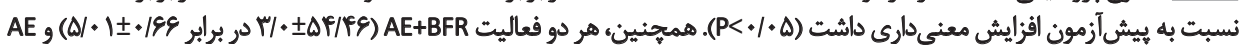

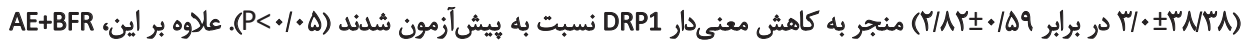

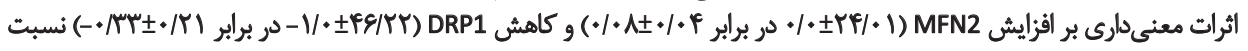

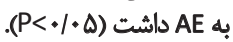

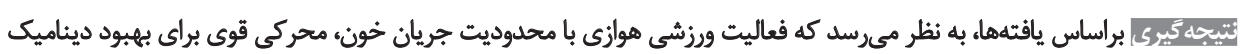

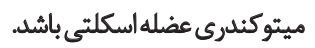

:Lotglowls

فعاليت ورزشي هوازي، محدوديت جريان خون، ديناميك ميتوكندرى، مجند، DRP1، MFN2
\end{abstract}

اسكلتى، كاهش ميزان پاحسازى متابوليتهاى توليدى و ايجاد

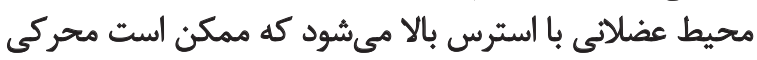

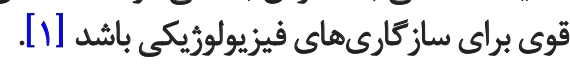

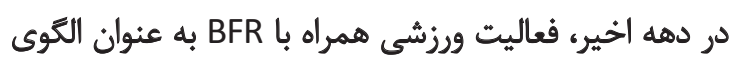

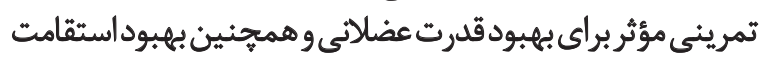

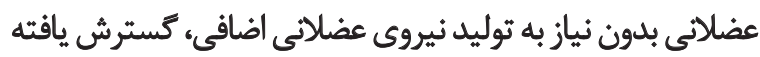

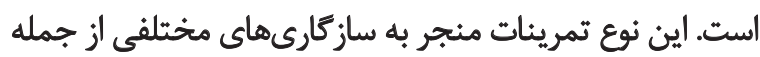

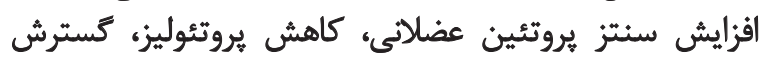

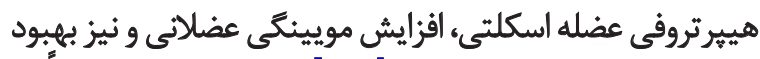
توان هوازى (VO

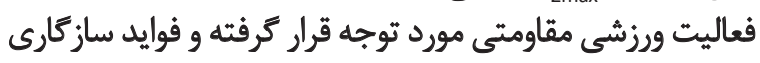

dale بلهاركيرى شيوههاى تمرينى جديد و مؤثر، همواره مورد

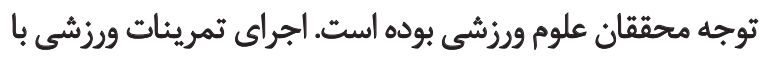

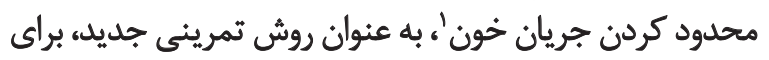

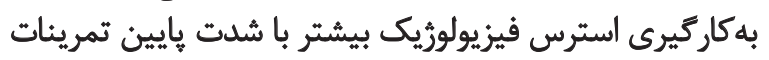

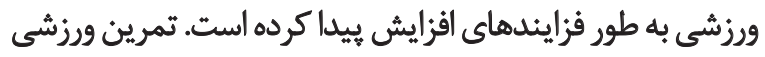

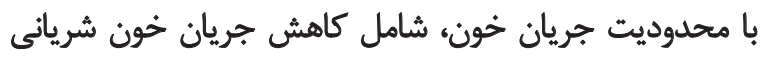

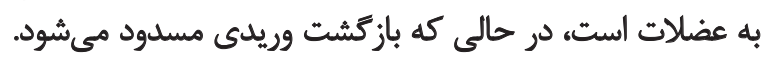

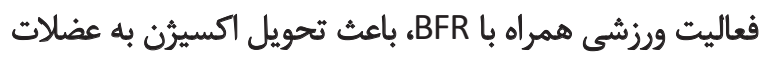

1. Blood Flow Restriction (BFR)

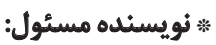
بهمن ميرزايى نويند نشانى: رشت، دانشكاه كيلان، دانشكده تربيت بلدنى و علوم ورزشيى

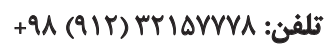
رايانامه: mirzaei@fila-wrestling.com 


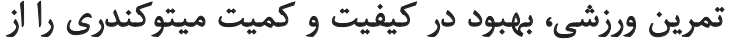

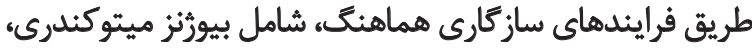

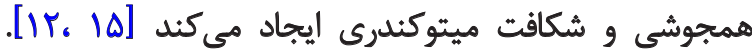

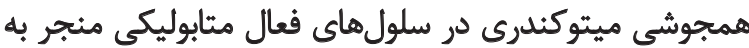

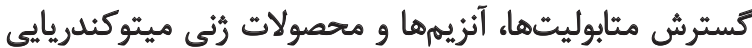

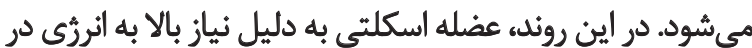

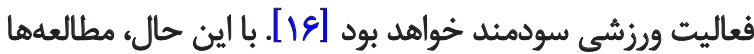

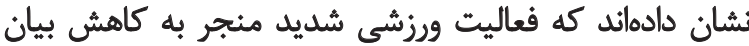
در عضله اسكلتي رتها تا MFN1,2

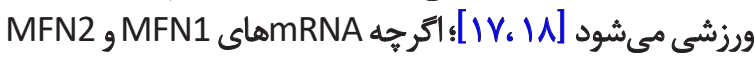

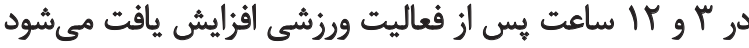

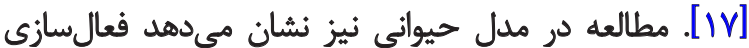

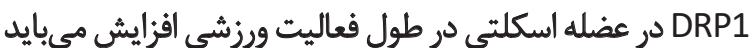
و تاواماندكى در سطح بالا باقى مي ماند [19 19].

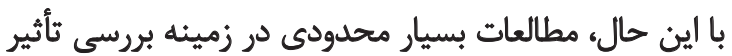

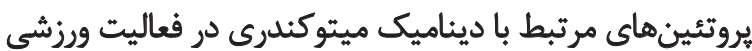

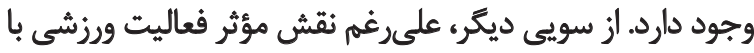

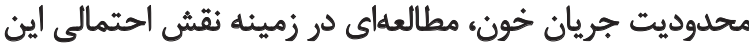

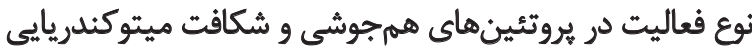

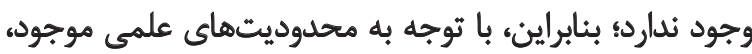

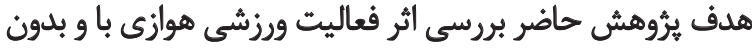

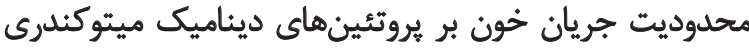
(DRP1 و Mضله اسكلتى انسان است.

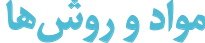

روش ئروهش حاضر نيمهتجربى با طرح بيشآزمون و و

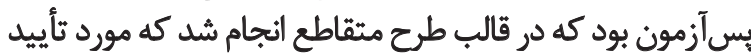

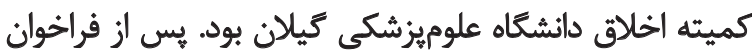

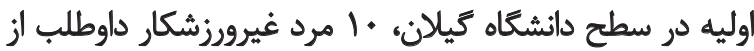

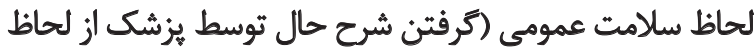

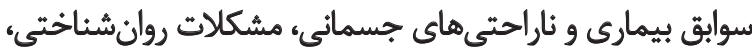

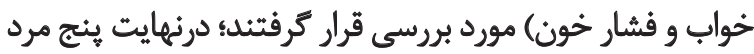

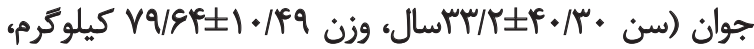

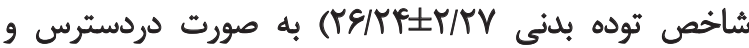

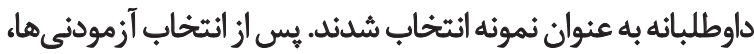

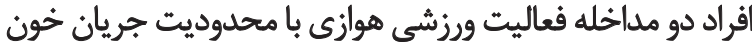

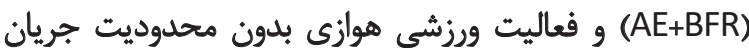
خون (AE) رادر دو روز مجزا اجرا كردند.

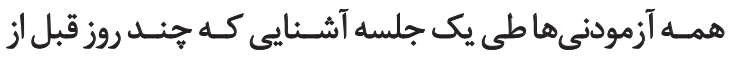

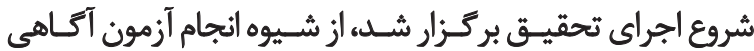

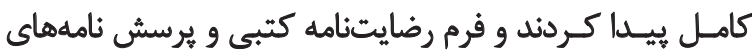

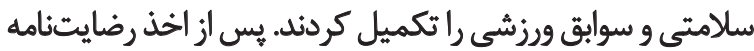

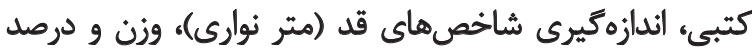

با اين نوع تمرينات، بلهخوبى درك شده است. با اين حال، تأثير

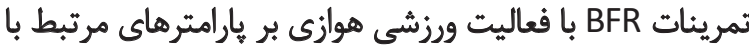

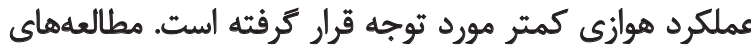

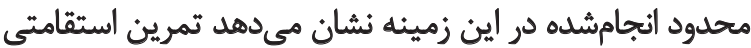

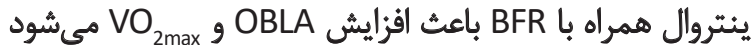

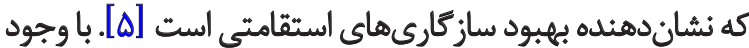

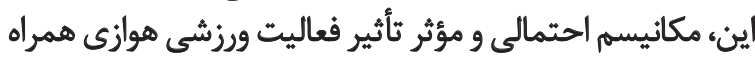

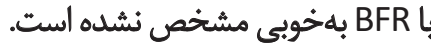

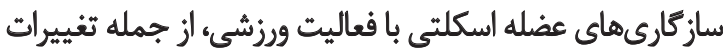

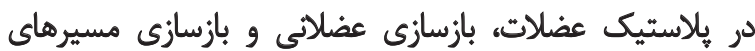

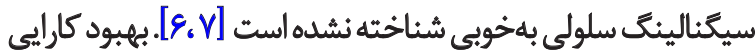

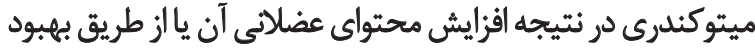

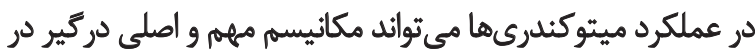

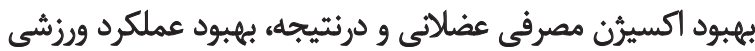

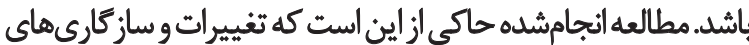

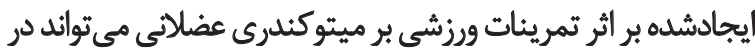

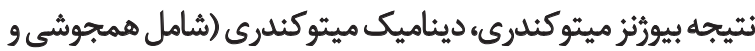
شكافت) و فرايند ميتوفارى اتفاق افتد [N] ].

همجوشى ميتوكندرى نقش مهمي در حفظ يكيارجكى

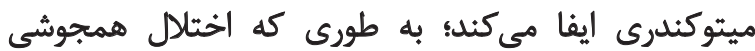

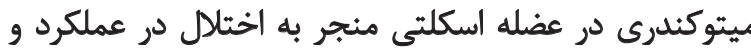

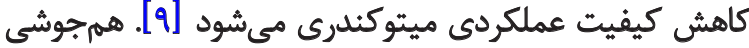

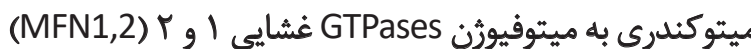
و إيتيك آتروفى (OPA-1) وابسته است. MFN-1,2 غشايى كاركردى هستند كه در غشاى خارئي

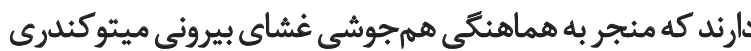

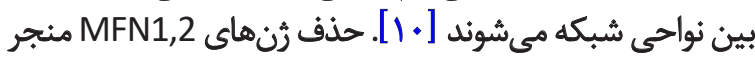

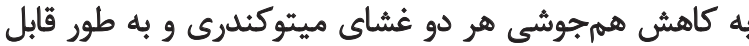

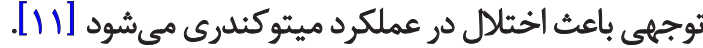
همجوشى ميتوكندرى متفاوت با شكافت ميتوكندرى

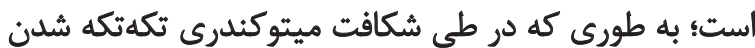

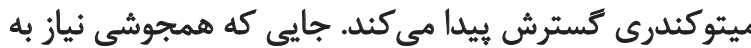

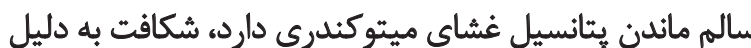

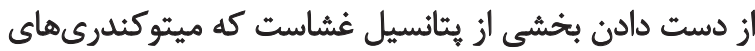

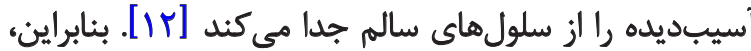
شكافت ميتوكندرى براى حفظ كيفيت ميتوكندرى ضرى ضرورى

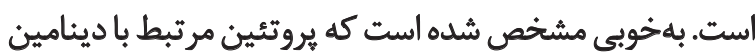

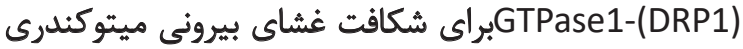

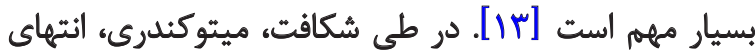

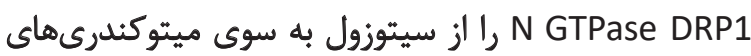

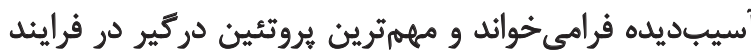

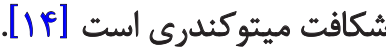


صورت ترفت. ميكروبيويسى يك روش كمتر تهاجمى است كه در

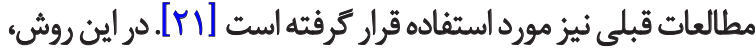

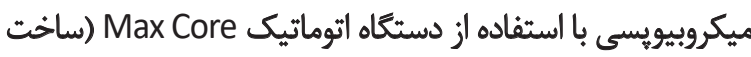

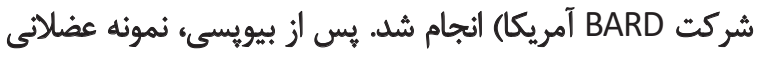

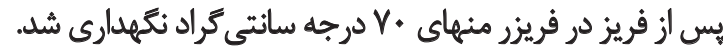
براى اندازهكيرى مقادير بروتئينى MFN2 و DRP1 از بادئ

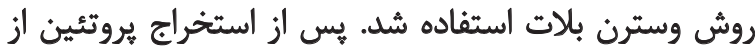

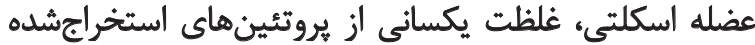

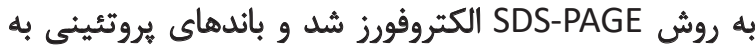

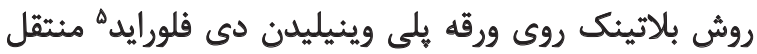

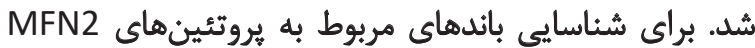

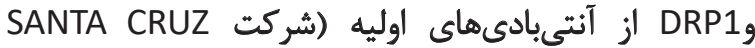

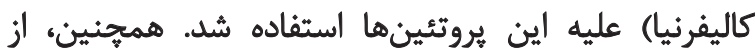

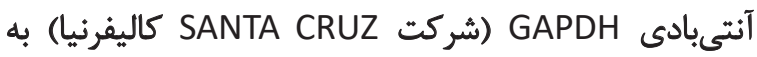

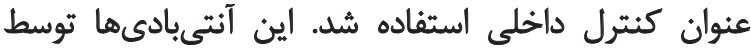

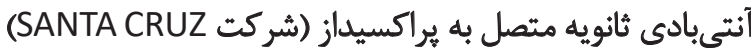

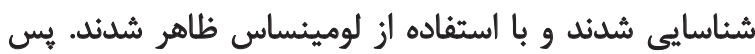

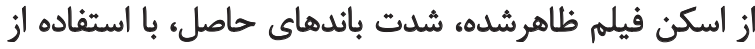

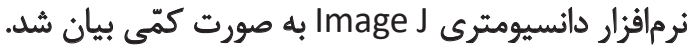

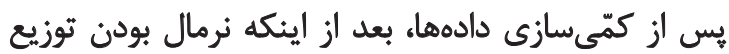

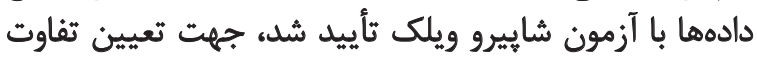

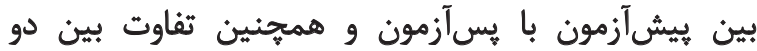

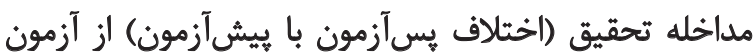

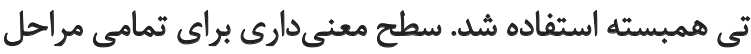

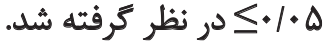

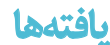

نتايج نشان داد هر دو فعاليت ورزشى هوازى با محدوديت

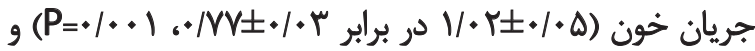

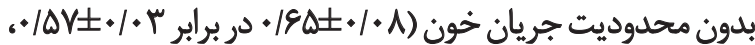
MFN2 منجر به افزايش معنى (P=॰

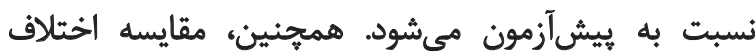

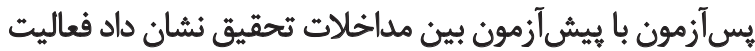

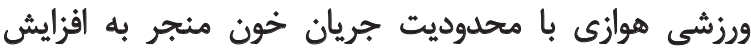

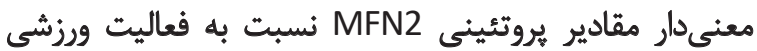

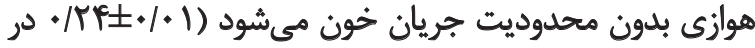

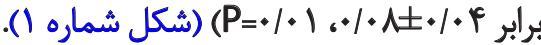

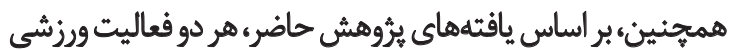

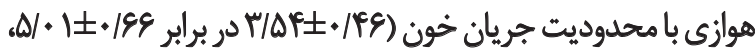

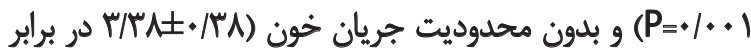

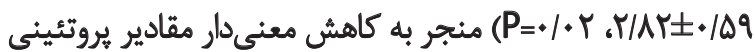

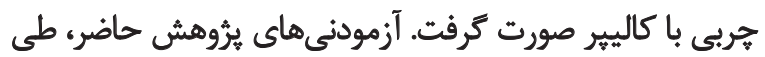

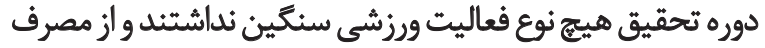

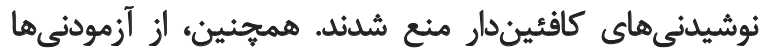

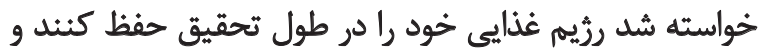

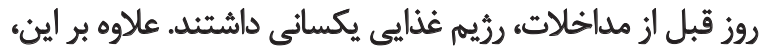

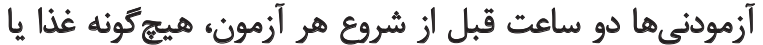
مايعاتى غير از آب مصرف نكردند. به منظور آشناسازى با نحسوه اجـراى مداخلات ورزشى،

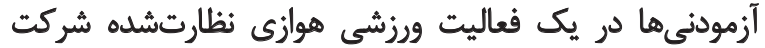

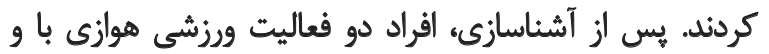

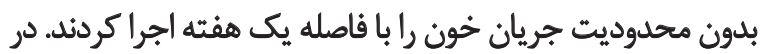

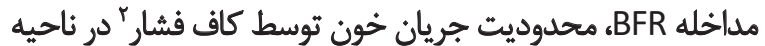

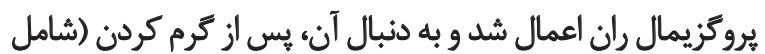

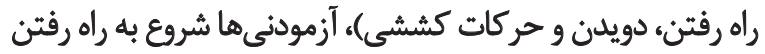

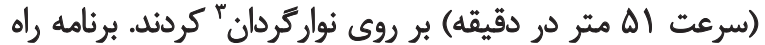

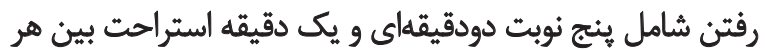

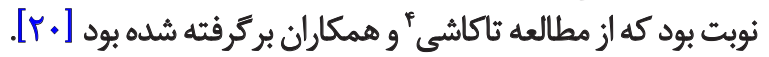

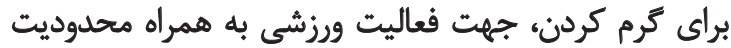

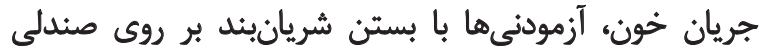

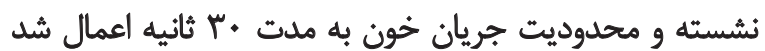

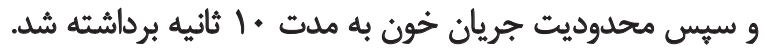

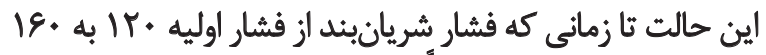

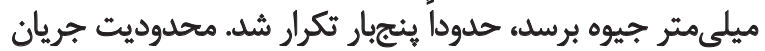

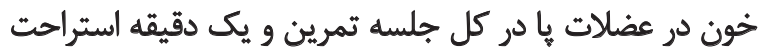

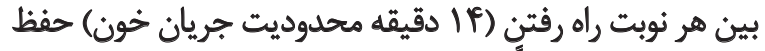

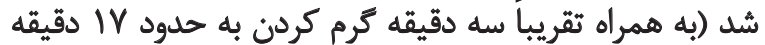

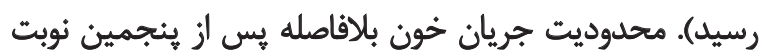

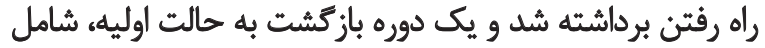

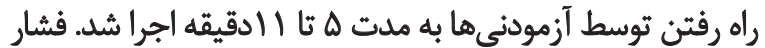

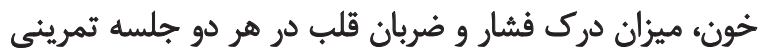

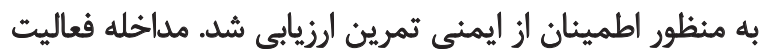

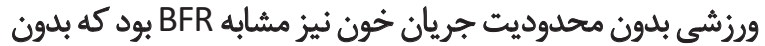

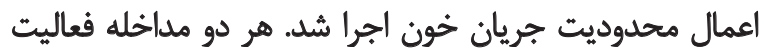

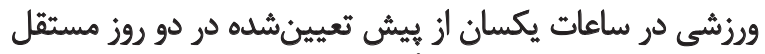

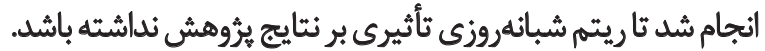
به منظور بررسى تغييرات MFN2 و DRP1، بيويسى از عضله

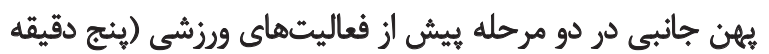

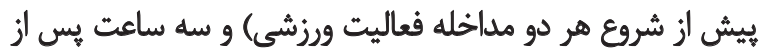

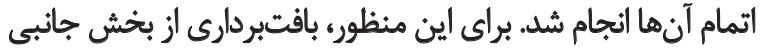

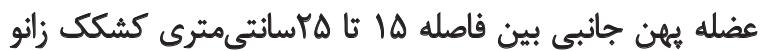

2. Pressure Cuff

3. Tredmill

4. Takashi 

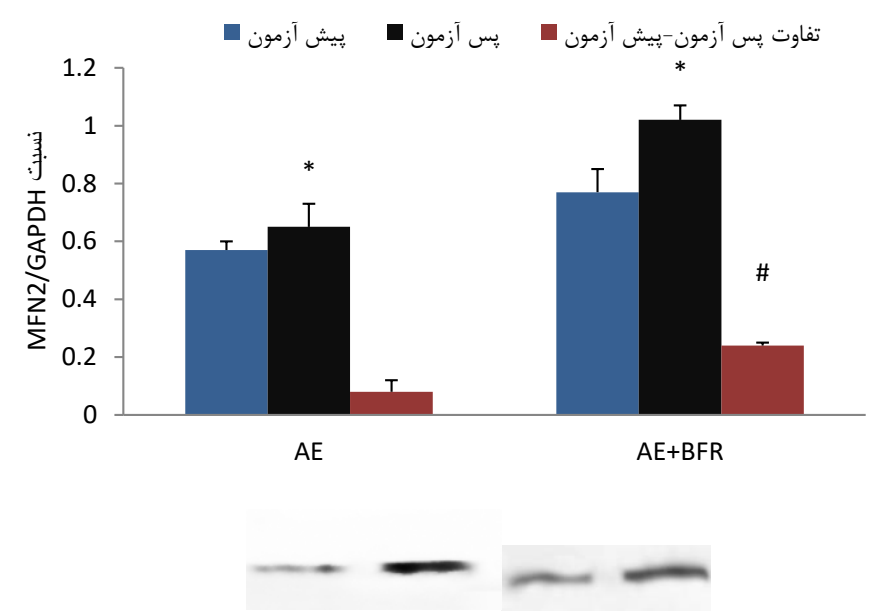

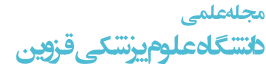

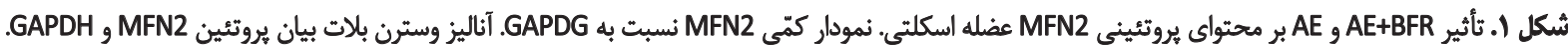

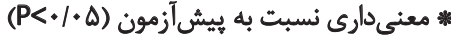

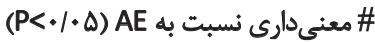

با همرجوشى ميتوكثدرى (MFN2) و كاهش بروتئين مرتبط با

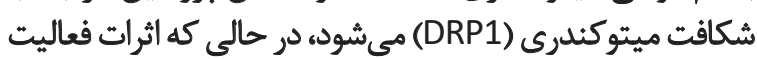

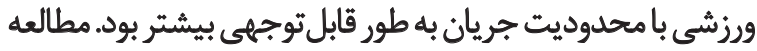

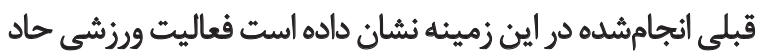

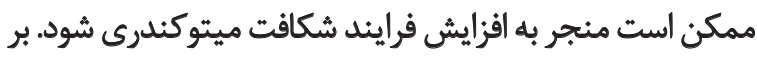

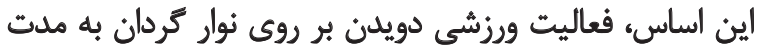

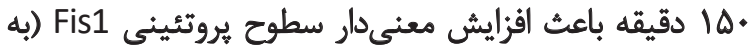

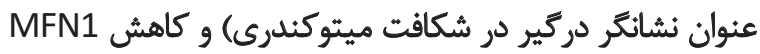

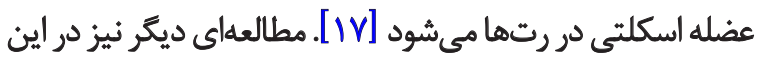

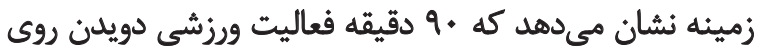

DRP1 نسبت به ييشآزمون شدند (شكل شماره Y).

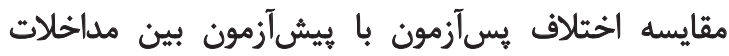

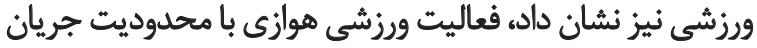

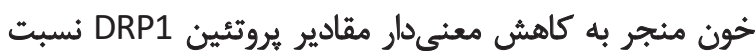

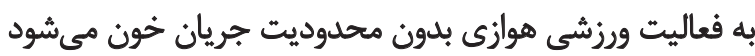

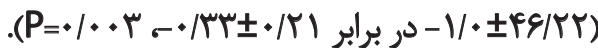

\section{بحث ونتيجه كَيرى}

يافتههاى يروهش حاضر نشان داد فعاليت ورزشى هوازى باو

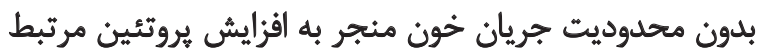
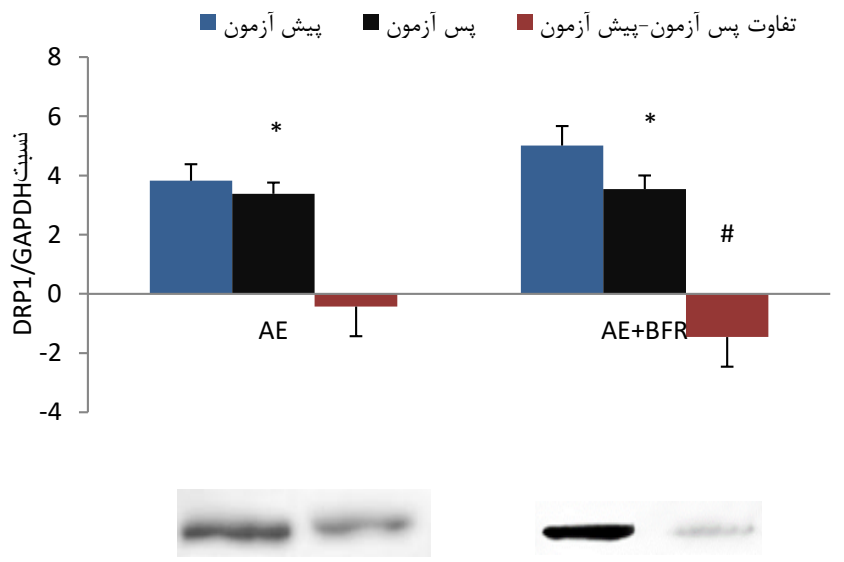

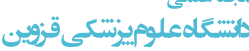

شكل Y. تأثير AE+BFR و AE بر محتواى بروتئيني DRP1 عضله اسكلتى. نمودار كتى DRP1 نسبت به GAPDG. آناليز وسترن بلات بيان يروتئين DRP1 و (P< /.A (APDH

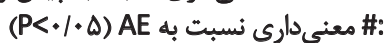


داد فعاليت ورزشى با محدوديت جريان خون اثرات قابلتوجهيى

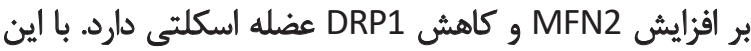

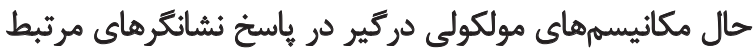

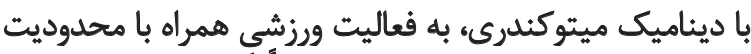

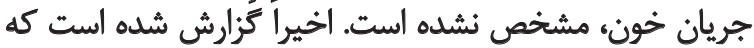

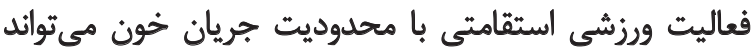

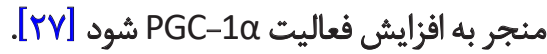
برخى نشانكر ها و واسطههاي سلولى در نتيجه فعاليت ورزشى

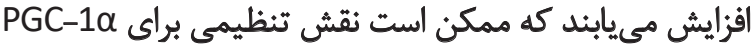

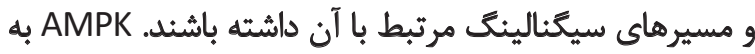

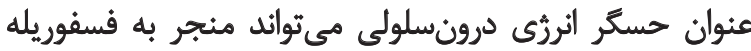

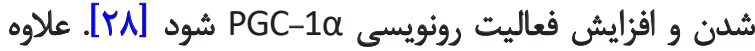

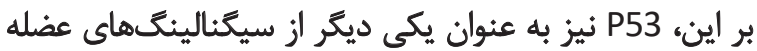

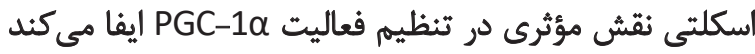

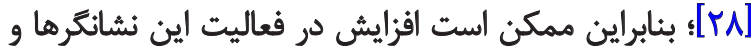

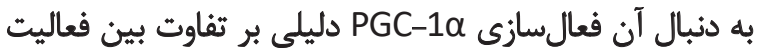

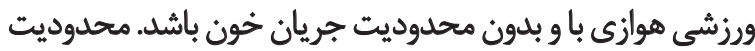
جريان خون و ايسكمى كه به دنبال فعاليت ورزشى بان با BFR اتفاق

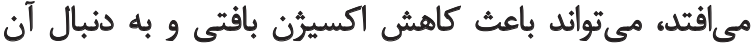

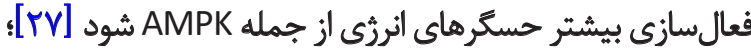

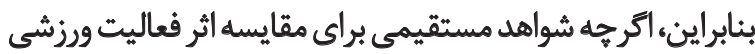

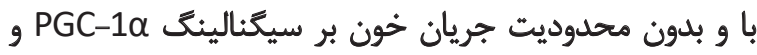

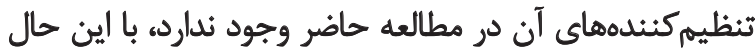

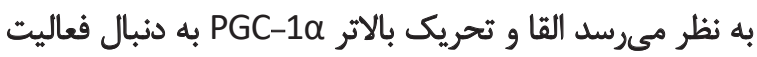

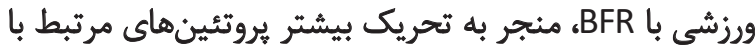
ديناميك ميتوكندرى شده است

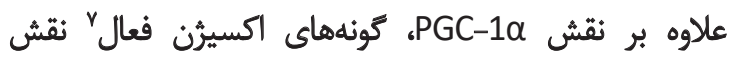

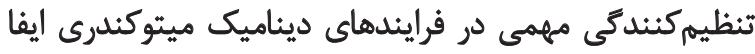

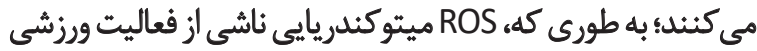

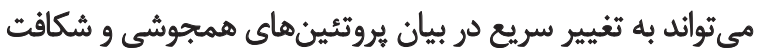

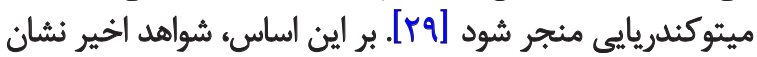

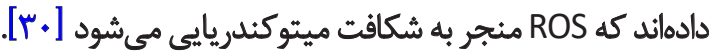

از سوى ديكر، مشخص شده است كه فعاليت ورزشى با بانداني

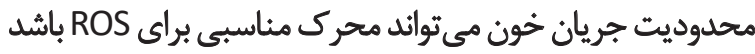

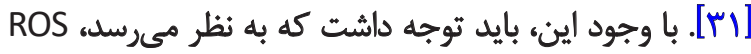

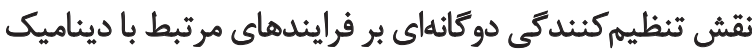

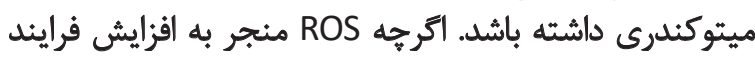

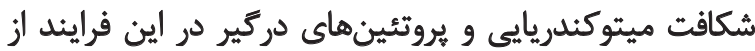

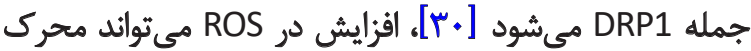

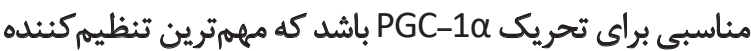

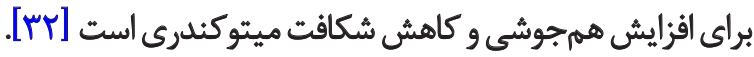

نواركردان باعث افزرايش فسفريلاسيون Drp1 و بدون تغييرات

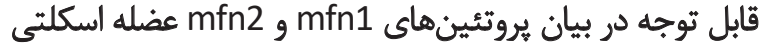

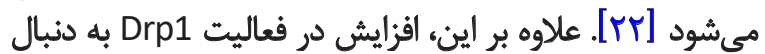

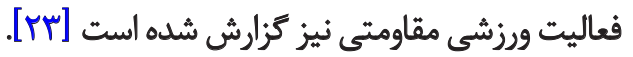
در مقابل، برخى ديكر از مطالعات انجامشده در اين زمينه

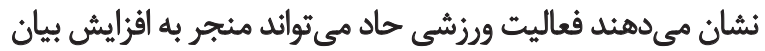

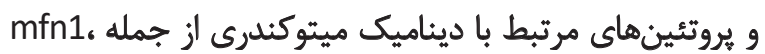

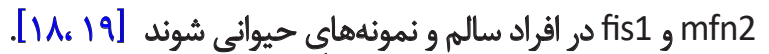

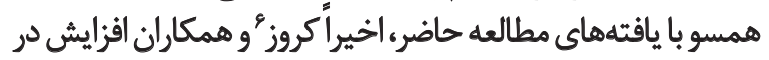

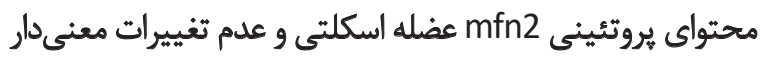

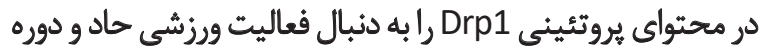

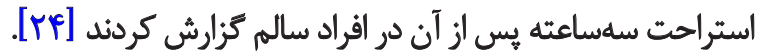

يافتههاي اين مطالعه نشان داد فعاليت ورزشى منجر به تسهيل

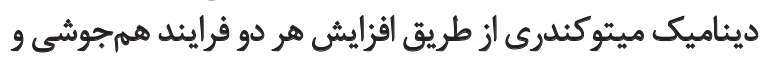

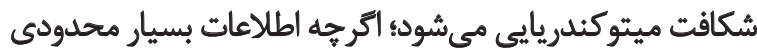

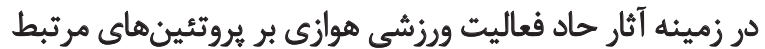

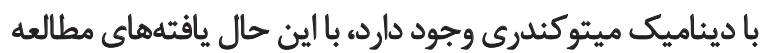

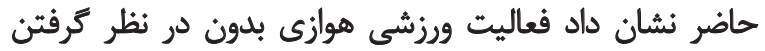

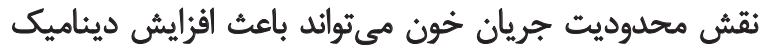

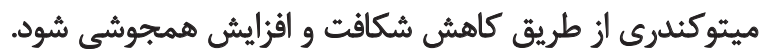

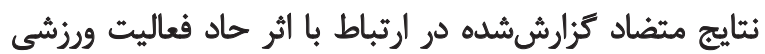

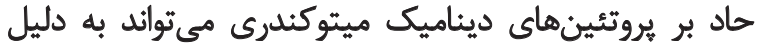

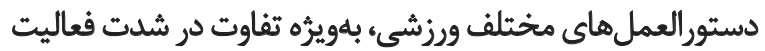
و همجنين زمان نمونهكيرى باشد.

مكانيسم ياسخ Drp1 و Mfn2 به فعاليت ورزشى حاد درك

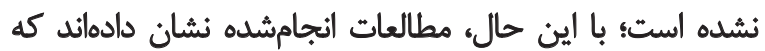

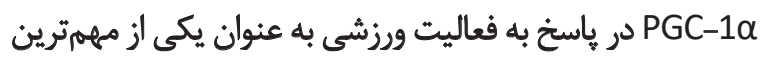

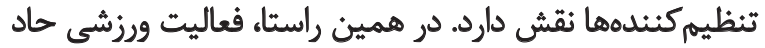

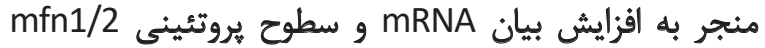
در عضله اسكلتى مىشود كه همراستا با افزايش

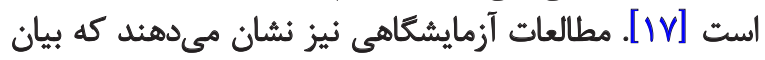

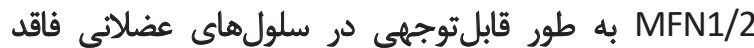

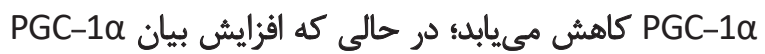

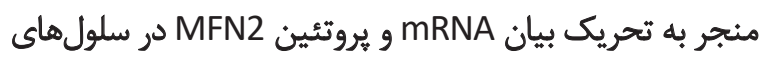

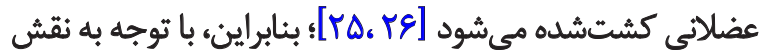

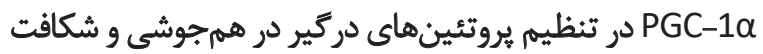

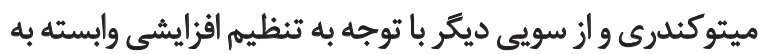

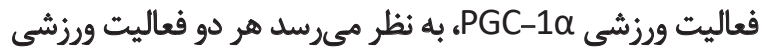

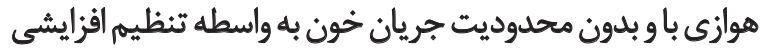

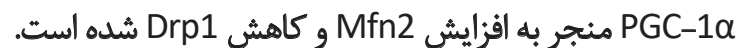
علاوه بر اين، براى اولينبار، مههمترين يافته يرؤهش حاضر نشان 
به نظر مىرسد كه نقش مOS مOS مئواند وابسته به مدتزمان

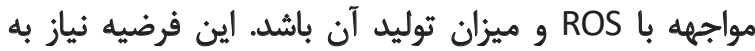

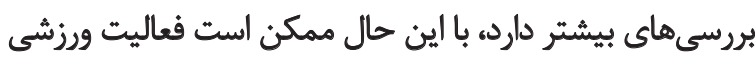

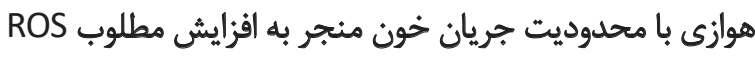

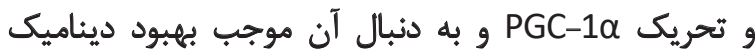
ميتوكندريايى، سه ساعت بيش از فعاليت ورزشى شده است

يافتههاى يروهش حاضر براى اولينبار نشان داد فعاليت ورزشى

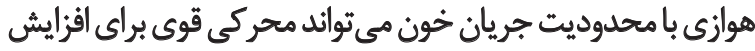

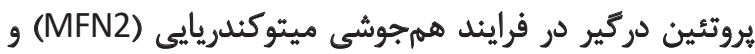
كاهش يروتئين دركير در شكافت ميتوكندرين فيني (DRP1) شود.

مهمترين محدوديتهاى يُروهش حاضر، عدم اندازهيرى

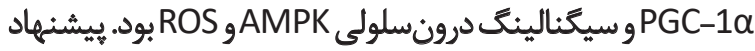

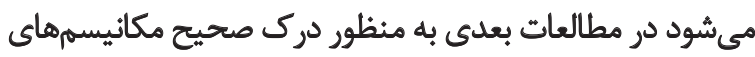

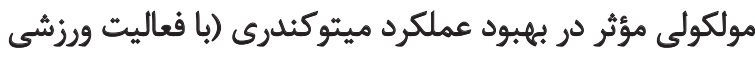

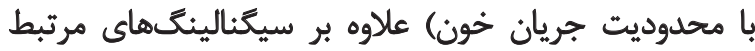

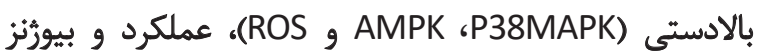

$$
\text { ميتوكندرى نيز بررسي شود. }
$$

مالاحظات اخلاقثى

$$
\text { ييروى ازٔ أصول اخلاق يثوهش }
$$

اين مطالعه در كميته اخلاق دانشَّاه علوميرِشكى كيلان باكد IR.GUMS.REC.1397.061 تأييد شده است.

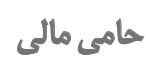

مقاله يُوهشى حاضر، مستخرج از رساله دكثرى تخصصى

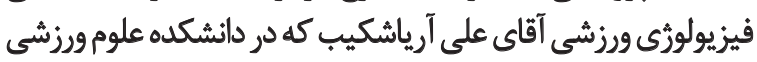

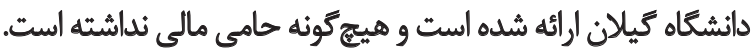

$$
\text { مشاركت نويسندكًان }
$$

مفهومسازي و اعتبارسنجى: بهمن ميرزايى و على آرياشكيب؛

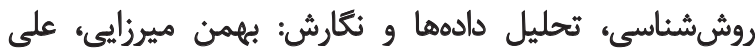

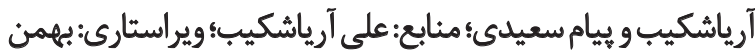

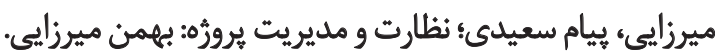

$$
\text { تعارض مناقع }
$$

هيج كونه تضاد منافعى از سوى نويسندكان كزارش نشد. 


\section{References}

[1] Paton CD, Addis SM, Taylor LA. The effects of muscle blood flow restriction during running training on measures of aerobic capacity and run time to exhaustion. Eur J Appl Physiol. 2017; 117(12):2579-85. [DOI:10.1007/s00421-017-3745-3] [PMID]

[2] Laurentino GC, Ugrinowitsch C, Roschel H, Aoki MS, Soares AG, Neves Jr M, et al. Strength training with blood flow restriction diminishes myostatin gene expression. Med Sci Sports Exerc. 2012; 44(3):406-12. [DOI:10.1249/MSS.0b013e318233b4bc] [PMID]

[3] Ozaki H, Kakigi R, Kobayashi H, Loenneke JP, Abe T, Naito H. Effects of walking combined with restricted leg blood flow on mTOR and MAPK signalling in young men. Acta Physiol (Oxf). 2014; 211(1):97-106. [DOI:10.1111/apha.12243] [PMID]

[4] Larkin KA, MacNeil RG, Dirain M, Sandesara B, Manini TM, Buford TW. Blood flow restriction enhances post-resistance exercise angiogenic gene expression. Med Sci Sports Exerc. 2012; 44(11):207783. [DOI:10.1249/MSS.0b013e3182625928] [PMID] [PMCID]

[5] de Oliveira MFM, Caputo F, Corvino RB, Denadai BS. Short-term low-intensity blood flow restricted interval training improves both aerobic fitness and muscle strength. Scand J Med Sci Sports. 2016; 26(9):1017:25. [DOI:10.1111/sms.12540] [PMID]

[6] Gundersen K. Excitation-transcription coupling in skeletal muscle: The molecular pathways of exercise. Biol Rev camb Philos Soc. 2011; 86(3):564-600. [DOI:10.1111/j.1469185X.2010.00161.x] [PMID] [PMCID]

[7] Ferraro E, Giammarioli AM, Chiandotto S, Spoletini I, Rosano G. Exercise-induced skeletal muscle remodeling and metabolic adaptation: Redox signaling and role of autophagy. Antioxid Redox Signal. 2014; 21(1):154-76. [DOI:10.1089/ ars.2013.5773] [PMID] [PMCID]

[8] Gottlieb RA, Carreira RS. Autophagy in health and disease. 5. Mitophagy as a way of life. Am J Physiol Cell Physiol. 2010; 299(2):C20310. [DOI:10.1152/ajpcell.00097.2010] [PMID] [PMCID]

[9] Eisner V, Lenaers G, Hajnóczky G. Mitochondrial fusion is frequent in skeletal muscle and supports excitation-contraction coupling. J Cell Biol. 2014; 205(2):179-95. [DOI:10.1083/ jcb.201312066] [PMID] [PMCID]

[10] Chen H, Chomyn A, Chan DC. Disruption of fusion results in mitochondrial heterogeneity and dysfunction. J Biol Chem. 2005; 280(28):26185-92. [DOI:10.1074/jbc.M503062200] [PMID]

[11] Caffin F, Prola A, Piquereau J, Novotova M, David DJ, Garnier $A$, et al. Altered skeletal muscle mitochondrial biogenesis but improved endurance capacity in trained OPA1-deficient mice. J physiol. 2013; 591(23):6017-37. [DOI:10.1113/jphysit ol.2013.263079] [PMID] [PMCID]

[12] Youle RJ, van der Bliek AM. Mitochondrial fission, fusion, and stress. Science. 2012; 337(6098):1062-5 [DOI:10.1126/scit ence.1219855] [PMID] [PMCID]

[13] Smirnova E, Shurland DL, Ryazantsev SN, van der Bliek AM. A human dynamin-related protein controls the distribution of mitochondria. J Cell Biol. 1998; 143(2):351-8. [DOI:10.1083/ jcb.143.2.351] [PMID] [PMCID]
[14] James DI, Parone PA, Mattenberger Y, Martinou JC. hFis1, a novel component of the mammalian mitochondrial fission machinery. J Biol Chem. 2003; 278(38):36373-9. [DOI:10.1074/ jbc.M303758200] [PMID]

[15] Miller BF, Hamilton KL. A perspective on the determination of mitochondrial biogenesis. Am J Physiol Endocrinol Metab. 2012; 302(5):E496-E9. [DOI:10.1152/ajpendo.00578.2011] [PMID] [PMCID]

[16] Westermann B. Bioenergetic role of mitochondrial fusion and fission. Biochim Biophys Acta. 2012; 1817(10):1833-8 [DOI:10.1016/j.bbabio.2012.02.033] [PMID]

[17] Ding H, Jiang N, Liu H, Liu X, Liu D, Zhao F, et al. Response of mitochondrial fusion and fission protein gene expression to exercise in rat skeletal muscle. Biochim Biophys Acta. 2010; 1800(3):250-6. [DOI:10.1016/j.bbagen.2009.08.007] [PMID]

[18] Cartoni R, Léger B, Hock MB, Praz M, Crettenand A, Pich S, et al. Mitofusins $1 / 2$ and ERR $\alpha$ expression are increased in human skeletal muscle after physical exercise. J physiol. 2005; 567(Pt 1):349-58. [DOI:10.1113/jphysiol.2005.092031] [PMID] [PMCID]

[19] Pagano AF, Py G, Bernardi H, Candau RB, Sanchez AMJ. Autophagy and protein turnover signaling in slow-twitch muscle during exercise. Med Sci Sports Exerc. 2014; 46(7):1314-25. [DOI:10.1249/MSS.0000000000000237] [PMID]

[20] Abe T, Sakamaki M, Fujita S, Ozaki H, Sugaya M, Sato Y, et al. Effects of low-intensity walk training with restricted leg blood flow on muscle strength and aerobic capacity in older adults. J Geriatr Phys Ther. 2010; 33(1):34-40. [PMID]

[21] Hayot M, Michaud A, Koechlin C, Caron MA, Leblanc P, Préfaut $C$, et al. Skeletal muscle microbiopsy: A validation study of a minimally invasive technique. Eur Respir J. 2005; 25(3):431 40. [DOI:10.1183/09031936.05.00053404] [PMID]

[22] Jamart C, Naslain D, Gilson H, Francaux M. Higher activation of autophagy in skeletal muscle of mice during endurance exercise in the fasted state. Am J Physiology Endocrinolo Metab. 2013; 305(8):E964-E74. [DOI:10.1152/ajpendo.00270.2013] [PMID]

[23] Kitaoka Y, Ogasawara R, Tamura Y, Fujita S, Hatta H. Effect of electrical stimulation-induced resistance exercise on $\mathrm{mi}$ tochondrial fission and fusion proteins in rat skeletal muscle. Appl Physiol Nutr Metab. 2015; 40(11):1137-42. [DOI:10.1139/ apnm-2015-0184] [PMID]

[24] Kruse R, Pedersen AJT, Kristensen JM, Petersson SJ, Wojtaszewski JFP, Højlund K. Intact initiation of autophagy and mitochondrial fission by acute exercise in skeletal muscle of patients with type 2 diabetes. Clini Sci (Lond). 2017; 131(1):3747. [DOI:10.1042/CS20160736] [PMID]

[25] St-Pierre J, Drori S, Uldry M, Silvaggi JM, Rhee J, Jäger S, et al. Suppression of reactive oxygen species and neurodegeneration by the PGC-1 transcriptional coactivators. Cell. 2006; 127(2):397-408. [DOI:10.1016/j.cell.2006.09.024] [PMID]

[26] Liesa M, Borda-d’Água B, Medina-Gómez G, Lelliott CJ, Paz JC, Rojo $M$, et al. Mitochondrial fusion is increased by the nuclear coactivator PGC-1ß. PLoS One. 2008; 3(10):e3613. [DOI:10.1371/journal.pone.0003613] [PMID] [PMCID] 
[27] Bahreinipour MA, Joukar S, Hovanloo F, Najafipour H, Naderi $\mathrm{V}$, Rajiamirhasani AR, et al. Mild aerobic training with blood flow restriction increases the hypertrophy index and MuSK in both slow and fast muscles of old rats: Role of PGC-1 $\alpha$. Life Sci. 2018; 202:103-9. [DOI:10.1016/j.Ifs.2018.03.051] [PMID]

[28] Hawley JA, Hargreaves M, Joyner MJ, Zierath JR. Integrative biology of exercise. Cell. 2014; 159(4):738-49. [DOI:10.1016/j. cell.2014.10.029] [PMID]

[29] Yu T, Robotham JL, Yoon Y. Increased production of reactive oxygen species in hyperglycemic conditions requires dynamic change of mitochondrial morphology. Proc Natl Acad Sci. 2006; 103(8):2653-8. [DOI:10.1073/pnas.0511154103] [PMID] [PMCID]

[30] Gomez-Lazaro M, Bonekamp NA, Galindo MF, Jordán J, Schrader M. 6-Hydroxydopamine (6-OHDA) induces Drp1dependent mitochondrial fragmentation in $\mathrm{SH}-\mathrm{SY} 5 \mathrm{Y}$ cells. Free Radic Biol Med. 2008; 44(11):1960-9. [DOI:10.1016/j.freerade biomed.2008.03.009] [PMID]

[31] Christiansen D, Murphy RM, Bangsbo J, Stathis CG, Bishop DJ. Increased FXYD1 and PGC-1 $\alpha$ mRNA after blood flow-restricted running is related to fibre type-specific AMPK signalling and oxidative stress in human muscle. Acta Physiol (Oxf). 2018; 223(2):e13045. [DOI:10.1111/apha.13045] [PMID] [PMCID]

[32] Irrcher I, Ljubicic V, Hood DA. Interactions between ROS and AMP kinase activity in the regulation of PGC-1 $\alpha$ transcription in skeletal muscle cells. Am J Physiol Cell Physiol. 2009; 296(1):C116-C23. [DOI:10.1152/ajpcell.00267.2007] [PMID] 
This Page Intentionally Left Blank 